\title{
On the Solvability of Convolution Equations in Beurling's Distributions
}

By

\author{
Dae Hyeon PaHK ${ }^{*}$ and Byung Keun SoHN**
}

\begin{abstract}
Let $\mathscr{D}_{w}^{\prime}$ be the space of Beurling's generalized distributions on $\mathbf{R}^{n}$ and $\mathscr{E}_{w}^{\prime}$ the spaces of generalized distributions which has compact support. We show that, for $S \in \mathscr{E}_{w}^{\prime}, S * \mathscr{D}_{w}^{\prime}$ $=\mathscr{D}_{w}^{\prime}$ is equivalent to the following: Every generalized distribution $u \in \mathscr{E}_{w}^{\prime}$ with $S * u \in$ $\mathscr{D}_{w}$ is in $\mathscr{D}_{w}$.
\end{abstract}

S. Abdullah [1] proves that, for a generalized distribution $S$ having compact support, the followings are equivalent;

(a) There exists positive constants $A, C$ such that

$$
\sup _{\substack{\left.x \mid \leq A \omega(\xi) \\ x \in \mathbf{R}^{(}\right)}}|\hat{S}(x+\xi)| \geq C \exp (-A \omega(\xi)) \quad \xi \in \mathbf{R}^{n}
$$

(b) $S * \mathscr{D}_{w}^{\prime}=\mathscr{D}_{w}^{\prime}$.

In this paper we show another property which is equivalent to the above.

Before stating our main theorem we briefly introduce the space of generalized distributions of Beurling type and the related results which we need in this paper. For details we refer to [3].

We denote by $M_{c}$ the set of all real valued functions $w$ on $\mathbf{R}^{n}$ satisfying the following conditions;

(a) $0=w(0) \leq w(\xi+\eta) \leq w(\xi)+w(\eta), \quad \xi, \eta \in \mathbf{R}^{n}$

(B) $\int_{\mathbf{R}^{n}} \frac{w(\xi)}{(1+|\xi|)^{n+1}} d \xi<\infty$.

( $\gamma$ ) $w(\xi) \geq a+b \log (1+|\xi|)$ for some constants $a$ and $b>0$.

This research is partly supported by KOSEF, GARC and BSRI, Ministry of Education. Communicated by T. Kawai, May 12, 1995.

1991 Mathematics Subject Classifications: Primary 35D10, 35H05.

* Department of Mathematics, Yonsei University, Seoul 120-749, Korea

* * Department of Mathematics, Inje University, Kimhae 621-749, Korea 
(ס) $w(\xi)=\sigma(|\xi|)$ for an increasing concave function $\sigma$ on $[0, \infty)$. For example, $w(\xi)=\log (1+|\xi|)$ and $w(\xi)=|\xi|^{\frac{1}{d}}, d>1$, satisfy all conditions. Throughout this paper $\omega$ represents an element in $M_{c}$.

Let $\mathscr{D}_{w}$ be the set of all $\phi$ in $L^{1}\left(R^{n}\right)$ such that $\phi$ has compact support and

$$
\|\phi\|_{\lambda}^{(w)}=\int_{\mathbf{R}^{n}}|\hat{\phi}(\xi)| \exp (\lambda w(\xi)) d \xi<\infty \text { for every } \lambda>0 .
$$

The topology on this space is given by the inductive limit topology of the Frechet spaces $\mathscr{D}_{w}(K)$ induced by the above semi-norms where $K$ is compact set in $\mathbb{R}^{n}$. We denote by $\mathscr{E}_{w}$ the set of all complex valued function $\phi$ in $\mathbb{R}^{n}$ such that $\phi \phi$ is in $\mathscr{D}_{w}$ for every $\phi \in \mathscr{D}_{w}$. The dual space of $\mathscr{D}_{w}$ is denoted by $\mathscr{D}_{w}^{\prime}$ whose elements are called the generalized distributions because of $\mathscr{D}_{w}^{\prime} \supset \mathscr{D}^{\prime}$ by $(\gamma)$. The dual space $\mathscr{C}_{w}^{\prime}$ of $\mathscr{E}_{w}$ is the space of generalized distributions which have compact support. In particular, $\mathscr{D}_{w}^{\prime}$ is identical to $\mathscr{D}^{\prime}$ and $\mathscr{C}_{w}^{\prime}$ to $\mathscr{E}^{\prime}$ when $w(\xi)=$ $\log (1+|\xi|)$. If $v * \phi(x)=\left\langle v_{y}, \phi(x-y)\right\rangle$ and $\langle u * v, \phi\rangle=\langle v, \breve{u} * \phi\rangle$ for $u \in \mathscr{E}_{w}^{\prime}$, $v \in \mathscr{D}_{w}^{\prime}$ and $\phi \in \mathscr{D}_{w}$, it can be easily seen that $\mathscr{D}_{w}^{\prime} * \mathscr{D}_{w} \subset \mathscr{D}_{w}$ and $\mathscr{E}_{w}^{\prime} * \mathscr{D}_{w}^{\prime} \subset \mathscr{D}_{w}^{\prime}$. Because of later fact, $\mathscr{C}_{w}^{\prime}$ is called the space of convolution operators in $\mathscr{D}_{w}^{\prime}$. Most results in the classical distribution space can be extended to the generalized distribution spaces. For instance, the Paley-Wiener-Schwartz theorem can be extended as follows: Let $K$ be a compact convex set in $\mathbb{R}^{n}$ with support function $H$. The Fourier-Laplace transform of $\phi \in \mathscr{D}_{w}(K)$ is the entire function $\hat{\phi}(\zeta)$ on $\mathbb{C}^{n}$ such that, for each $\lambda>0$ and $\epsilon>0$, there exists a constant $C_{\lambda, \epsilon}$ satisfying

$$
|\hat{\phi}(\xi+i \eta)| \leq C_{\lambda, \epsilon} \exp (H(\eta)+\epsilon|\eta|-\lambda w(\xi)), \quad \zeta=\xi+i \eta \in \mathbb{C}^{n}
$$

and also the converse holds. Moreover, the Fourier-Laplace transform of $u \in \mathscr{E}_{w}^{\prime}$ with $\operatorname{supp}(u) \subset K$ is the entire function $\hat{u}(\zeta)$ in $\mathbb{C}^{n}$ such that, for each $\epsilon>0$, there exist $\lambda>0$ and $C$ satisfying

$$
|\hat{u}(\xi+i \eta)| \leq C \exp (H(\eta)+\epsilon|\eta|+\lambda w(\xi)), \quad \zeta=\xi+i \eta \in \mathbb{C}^{n}
$$

and also the converse holds.

We now state our main theorem.

Theorem. For $S \in \mathscr{E}_{w}^{\prime}$, the followings are equivalent:

(a) there exist positive constants $A, C$ such that

$$
\sup _{\substack{|x| \leq A w(\xi) \\ x \in \mathbb{R}^{n}}}|\hat{S}(x+\xi)| \geq C \exp (-A w(\xi)), \quad \xi \in \mathbb{R}^{n}
$$

(b) $S * \mathscr{D}_{w}^{\prime}=\mathscr{D}_{w}^{\prime}$.

(c) If $u \in \mathscr{E}_{w}^{\prime}$ and $S * u \in \mathscr{D}_{w}$, then $u \in \mathscr{D}_{w}$.

The equivalence of (a) and (b) is proved by S. Abdullah [1]. Taking $w(\xi)=\log (1+|\xi|)$, our result includes the Ehrenpreis result as a special case, but the proof is quite different from his one. Due to the definition of hypoelliptic 
convolution operator $S$, which is defined as follows:

every $u \in \mathscr{D}_{w}^{\prime}$ with $S * u \in \mathscr{E}_{w}$ is in $\mathscr{E}_{w}$,

every hypoelliptic convolution operator is solvable, which means 'satisfying (b)', according to (c). In [6] ([7], resp.) we also study the same problem in the space of distributions which grow no faster than $\exp \left(k|x|^{p}\right)(\exp (M(k x))$, resp.) for some $k>0$. We now start the proof of theorem.

Proof of Theorem. According to the above remark, it suffices to show that (b) $\Rightarrow$ (c) $\Rightarrow$ (d).

(b) $\Rightarrow$ (c) : Denoting by $T=\check{S}$, the mapping $S^{*}: u \rightarrow S * u$ of $\mathscr{D}_{w}^{\prime}$ into $\mathscr{D}_{w}^{\prime}$ is the transpose of the mapping $T^{*}: \phi \rightarrow T * \phi$ of $\mathscr{D}_{w}$ into $\mathscr{D}_{w}$. Then the condition (b) is satisfied if and only if $T^{*}$ is an isomorphism of $\mathscr{D}_{w}$ onto $T * \mathscr{D}_{w}$ (see e.g., Corollary on pp.92 of [4]). In particular, the inverse mapping $T * \phi \rightarrow \phi$ of $T * \mathscr{D}_{w}$ onto $\mathscr{D}_{w}$ is continuous.

Suppose now that $S * u=\phi$, where $u \in \mathscr{E}_{w}^{\prime}$ and $\phi \in \mathscr{D}_{w}$. Then

$$
T * \check{u}=\check{\phi}
$$

and so it suffices to show that $\check{u}$ is in $\mathscr{D}_{w}$. Choosing $\phi \in \mathscr{D}_{w}$ with $\operatorname{supp}(\phi) \subset$ $B(0,1)=\left\{x \in \mathbb{R}^{n}:|x| \leq 1\right\}$ and $\hat{\phi}(0)=1$, we have a sequence $\left\{\phi_{k}(x)=\right.$ $k^{n} \psi(k x\}_{k=1}^{\infty}$ of approximate identities in $\mathscr{D}_{w}$. Acting $\phi_{k}$ to the both side of (1), we have

$$
T *\left(\check{u} * \phi_{k}\right)=\phi * \phi_{k}
$$

and the convolution $\check{u} * \phi_{k}$ and $\check{\phi} * \phi_{k}$ are in $\mathscr{D}_{w}$. Moreover, the sequence $\left\{\phi_{k}\right\}$ converges in $\mathscr{E}_{w}^{\prime}$ to $\delta$, the Dirac measure at the origin. Hence $\check{\phi} * \phi_{k} \rightarrow \check{\phi}$ in $\mathscr{D}_{w}$ and $\check{u} * \phi_{k} \rightarrow \check{u}$ in $\mathscr{E}_{w}^{\prime}$. On the other hand, the sequence $\left\{\check{u} * \phi_{k}\right\}$ converges in $\mathscr{D}_{w}$, by the continuity of the inverse mapping of $T^{*}$. The limit must be again $\check{u}$, and so $\check{u}$ is a function in $\mathscr{D}_{w}$.

(c) $\Rightarrow$ (a): Let $K$ be the closed unit ball in $\mathbf{R}^{n}$ and let $\mathscr{F}$ be the set of all functions $u$ in $C\left(\mathbf{R}^{n}\right)$ such that $\operatorname{supp}(u) \subset K$ and $S * u \in \mathscr{D}_{w}$. We provide $\mathscr{F}$ with the topology defined by the seminorms

$$
\text { i } u\left\|_{k}=\right\| u\left\|_{\infty}+\right\| S * u \|_{k}^{(w)}, \quad k=1,2, \cdots,
$$

where $\left|u \|_{\infty_{\infty}}=\sup _{x \in \mathbb{R}^{n}}\right| u(x) \mid$. Then $\mathscr{F}$ becomes a Frechet space. Furthermore, let $\mathscr{G}$ be the space of all functions $u \in C^{1}\left(\mathbf{R}^{n}\right)$ such that $\operatorname{supp}(u) \subset K$ and

$$
\|u\|=\sup _{x \in K,|\alpha| \leq 1}\left|D^{\alpha} u(x)\right|<\infty .
$$

Then $\mathscr{G}$ becomes a Banach space under this norm.

By the assumption (c), each function $u \in \mathscr{F}$ is in $\mathscr{G}$. Also the natural mapping $\mathscr{F} \hookrightarrow \mathscr{G}$ is closed and therefore continuous. Consequently, there exist an integer $k$ and a constant $C_{1}$ such that 


$$
\|u\| \leq C_{1}\left(\|u\|_{\infty}+\|S * u\|_{k}^{(w)}\right)
$$

for all $u \in \mathscr{F}$, which gives

$$
\|u\|-C_{1}\|u\|_{\infty} \leq C_{1}\|S * u\|_{k}^{(w)} .
$$

On the other hand, by $(\gamma)$ we have

$$
\begin{aligned}
\|S * u\|_{k}^{(w)}= & \int_{\mathbb{R}^{n}} \widehat{S * u}(\xi) \mid \exp (k w(\xi)) d \xi \\
\leq & \left(\sup _{\mathbf{R}^{n}} \exp \left(\left(k+\frac{n+1}{b}\right) w(\xi)\right)|\widehat{S * u}(\xi)|\right) \\
& \quad \cdot \int_{\mathbf{R}^{n}} \exp \left(-\frac{n+1}{b} w(\xi)\right) d \xi \\
\leq & C_{2}\left(\sup _{\mathbb{R}^{n}} \exp \left(\left(k+\frac{n+1}{b}\right) w(\xi)\right)|\widehat{S * u}(\xi)|\right) .
\end{aligned}
$$

Substituting this inequality into (6), we have

$$
\|u\|-C_{1}\|u\|_{\infty} \leq C \sup _{\mathbb{R}^{2}} \exp (\mu w(\xi))|\widehat{S * u}(\xi)|
$$

for every $u \in \mathscr{F}$, where $C=C_{1} C_{2}$ and $\mu=k+\frac{n+1}{b}$.

We notice that the condition (a) is implied by the following condition: there exists a constant $s_{1}>0$ such that for all $\xi \in \mathbf{R}^{n}$ with $|\xi| \geq s_{1}$,

$$
\sup _{|x| \leq A w(\xi)}|\hat{S}(x+\xi)| \geq \exp (-A w(\xi)),
$$

for some $A>0$.

Suppose now that the condition (a) does not hold. By the above implication, it follows that for any $j=1,2, \cdots$, there exists $\xi_{j} \in \mathbb{R}^{n}$ with $\left|\xi_{j}\right| \rightarrow \infty$ as $j \rightarrow \infty$ and

$$
\sup _{|x| \leq j w\left(\xi_{j}\right)}\left|\hat{S}\left(x+\xi_{j}\right)\right|<\exp \left(-j w\left(\xi_{j}\right)\right) .
$$

Choose $\phi \in \mathscr{D}_{w}$ such that $\phi \geq 0, \operatorname{supp}(\phi) \subset K$ and $\hat{\phi}(0)=1$. We define the function $\phi_{j}$ by

$$
\phi_{j}(x)=\exp \left(i<x, \xi_{j}>\right) \underbrace{\phi_{k,} * \cdots * \phi_{k}}_{k, \text { times }},
$$

where $\phi_{k_{j}}(x)=k_{j}^{n} \phi\left(k_{j} x\right)$ and $k_{j}$ is the Gaussian integer of $w\left(\xi_{j}\right)$. Then $\phi_{j} \in$ $\mathscr{D}_{w}(K)$ with $\int\left|\phi_{j}(x)\right| d x=1$ and so $\phi_{j} \in \mathscr{F}$.

Substituting $\phi_{j}$ 's into the inequality (7), we claim that the left side of (7) goes to $\infty$ and the right to 0 , as $j \rightarrow \infty$, which gives the desired contradiction.

To see this, we first estimate 


$$
\left\|\phi_{j}\right\|=\sup _{\substack{x \in \mathbb{R}^{n} \\|\alpha| \leq 1}}\left|D^{\alpha} \phi_{j}(x) \geq \frac{\left|\xi_{j}\right|}{n} \sup _{x \in \mathbb{R}^{n}}\right| \phi_{j}(x) \mid .
$$

Viewing $1=\int_{\mathbf{R}^{n}}\left|\phi_{j}(x)\right| d x \leq C_{2}\|\phi\|_{\infty}$, where $C_{2}$ is the volume of the ball $K$ in $\mathbf{R}^{n}$,

$$
\left\|\phi_{j}\right\|_{\infty} \geq \frac{1}{C_{2}}
$$

Substituting (10) and (11) into (7), the left-hand side of (7) tends to,

$$
\begin{aligned}
\lim _{j \rightarrow \infty}\left(\left\|\phi_{j}\right\|-C_{1}\left\|\phi_{j}\right\|_{\infty}\right) & \geq \lim _{j \rightarrow \infty}\left(\frac{\left|\xi_{j}\right|}{n}-C_{1}\right)\|\phi\|_{\infty} \\
& \geq \lim _{j \rightarrow \infty}\left(\frac{\left|\xi_{j}\right|}{n}-C_{1}\right) \frac{1}{C_{2}}=\infty .
\end{aligned}
$$

On the other hand, the right side of (7) can be written as

$$
\begin{aligned}
\sup _{\xi \in \mathbb{R}^{n}} \exp (\mu w(\xi))|\hat{S}(\xi)|\left|\hat{\phi}_{j}(\xi)\right| \\
=\sup _{|\xi-\xi,| \leq A_{1} w(\xi,)} \exp (\mu w(\xi))|\hat{S}(\xi)|\left|\hat{\phi}_{j}(\xi)\right| \\
\quad+\sup _{|\xi-\xi,|>A_{1} w(\xi)} \exp (\mu w(\xi)) ! \hat{S}(\xi) !\left|\hat{\phi}_{j}(\xi)\right|,
\end{aligned}
$$

for a fixed number $A_{1}$ which will be determined in the later.

From (8) and the facts that $\left|\hat{\phi}_{j}(\xi)\right| \leq \int\left|\phi_{j}(x)\right| d x=1$ and $w(\xi) \leq$ $M(1+|\xi|)$ for some $M$, the first term of (12) is bounded by

$$
\begin{aligned}
\sup _{|\xi-\xi,| \leq A_{2} w(\xi,)} \exp (\mu w(\xi)) & |\hat{S}(\xi)| \mid \hat{\phi}_{j}(\xi) i \\
\leq & +\sup _{|\xi| \leq A, w(\xi)} \exp \left(\mu w\left(\xi+\xi_{j}\right)\right)\left|\hat{S}\left(\xi+\xi_{j}\right)\right| \\
\leq & \exp \left(\mu\left(M+w\left(\xi_{j}\right)\right)\right) \sup _{|\xi| \leq A_{1} w\left(\xi_{,}\right)} \exp (\mu M|\xi|)\left|\hat{S}\left(\xi+\xi_{j}\right)\right| \\
\leq & \exp (\mu M) \exp \left(\left(\mu+A_{1} \mu M-j\right) w\left(\xi_{j}\right)\right) .
\end{aligned}
$$

Therefore the first term of (12) approaches to 0 as $j \rightarrow \infty$.

For the estimation of the second term of (12), using by the Paley-WienerSchwartz theorem for $S$ and $\phi_{j}$, we have that $|\hat{S}(\xi)| \leq C \exp (\lambda w(\xi))$ for some $\lambda>0$ and

$$
\left|\hat{\phi}_{j}(\xi)\right|=\left|\hat{\phi}\left(\frac{\xi-\xi_{j}}{k_{j}}\right)\right|^{k_{j}} \leq\left(C \exp \left(-(\mu+\lambda+1) w\left(\frac{\xi-\xi_{j}}{k_{j}}\right)\right)\right)^{k_{j}} .
$$

From this observation and the notation $d=\log C$, the second term of (12) is bounded by 


$$
\begin{aligned}
& \sup _{|\xi-\xi,|>A_{2} w(\xi)} \exp (\mu w(\xi))|\hat{S}(\xi)|\left|\hat{\phi}_{j}(\xi)\right| \\
& \leq C \sup _{|\xi-\xi,|>A_{1} w(\xi,)} \exp (\mu w(\xi)) \exp (\lambda w(\xi)) C^{k,} \\
& \cdot \exp \left(-(\mu+\lambda+1) k_{j} w\left(\frac{\xi-\xi_{j}}{k_{j}}\right)\right) \\
& \leq C \sup _{|\xi-\xi,|>A_{1} w(\xi)} \exp ((\mu+\lambda) w(\xi)) \exp \left(d w\left(\xi_{j}\right)\right) \\
& \cdot \exp \left(-(\mu+\lambda+1) k_{j} w\left(\frac{\xi-\xi_{j}}{k_{j}}\right)\right) \\
& \leq C \sup _{|\xi-\xi,|>A_{i} w(\xi)}\left\{\exp \left(k_{j}(\mu+\lambda) w\left(\frac{\xi-\xi_{j}}{k_{j}}\right)\right)\right. \\
& \cdot \exp \left((\mu+\lambda+d) w\left(\xi_{j}\right)\right) \\
& \left.\cdot \exp \left(-(\mu+\lambda+1) k_{j} w\left(\frac{\xi-\xi_{j}}{k_{j}}\right)\right)\right\} \\
& =C \sup _{\left|\xi-\xi_{,}\right|>A_{2} w(\xi,)} \exp \left(-k_{j} w\left(\frac{\xi-\xi_{j}}{k_{j}}\right)\right) \\
& \text { - } \exp \left((\mu+\lambda+d) w\left(\xi_{j}\right)\right) \\
& \leq C \exp \left(-k_{j} w\left(A_{1}\right)\right) \exp \left((\mu+\lambda+d) w\left(\xi_{j}\right)\right) \\
& \leq C \exp \left(w\left(A_{1}\right)\right) \exp \left(\left(\mu+\lambda+d-w\left(A_{1}\right)\right) w\left(\xi_{j}\right)\right) \text {. }
\end{aligned}
$$

Taking $A_{1}$ so large that $w\left(A_{1}\right)>\mu+\lambda+d$, the second term of (12) approaches to 0 as $j \rightarrow \infty$.

Combining both estimates the right side of (7) goes to 0 as $j \rightarrow \infty$, which we desired.

\section{References}

[1] Abdullah, S., Convolution equations in Beurling's distributions, Acta Math. Hung., 52 (1988), 7-20.

[2] Beurling, A., Quasi-analyticity and general distributions, Lectures 4 and 5, A.M.S. Summer Institute, Stanford, 1961 (mimeographed)

[3] Bjorck, G., Linear partial differential operators in the generalized distributions, Ark. Math., 6 (1966), 357-407.

[4] Dieudonné, J et Schwartz, L., La dualite dans les espaces (FF) et. (LLFF), Ann. Inst. Fourier Grenoble, 1 (1949), 61-101.

[5] Ehrenpreis, L., Solutions of some problems of division IV, Invertible and elliptic operators, Amer. J. Math., 82 (1960), 522-588.

[6] Pahk, D. H and Sohn, B. K., Relation between solvability and regularity of convolution operators in $\mathscr{K}_{p}^{\prime}, p>1$, J. Math. Anal. Appl., 185 (1994), 207-214.

[7] Pahk, D. H and Sohn, B. K., On the solvability of convolution equations in $\mathscr{K}_{M}^{\prime}$, to appear in Tsukuba, J. Math.

[8] Sznajder, $\mathrm{S}$ and Zielezny, Z., On some properties of convolution operators in $\mathscr{K}_{1}^{\prime}$ and S', J. Math. Anal. Appl., 65 (1978), 543-554. 